\title{
Updating of Episodic Memories Depends on the Strength of New Learning After Memory Reactivation
}

\author{
Sonja Wichert, Oliver T. Wolf, and Lars Schwabe \\ Ruhr-University Bochum, Bochum, Germany
}

\begin{abstract}
After reactivation, apparently stable memories may reenter an unstable state in which they are modifiable, requiring another phase of stabilization, called reconsolidation. Recent evidence shows that during reconsolidation, reactivated memories may be updated by the incorporation of new information. In the present study, we examined whether the updating of episodic memories depends on the strength of new encoding after reactivation. To this end, healthy participants learned negative and neutral pictures, reactivated them 1 week later, and learned new pictures either once or three times immediately after reactivation. A recognition test was performed another week later. Our results show that the impact of new learning after reactivation depended critically on the strength of new encoding: Whereas learning new pictures only once after reactivation had no effect on subsequent memory, learning new pictures three times after reactivation reduced subsequent memory accuracy, mainly due to intrusions from the newly learned pictures. Our findings indicate that the strength of new encoding after reactivation plays a critical role for the updating of episodic memories and may have important implications for therapeutic interventions that aim to alter unwanted memories after reactivation.
\end{abstract}

Keywords: reconsolidation, reactivation, new learning, episodic memory

Memories can be altered a long time after acquisition (Loftus \& Pickrell, 1995). This dynamic nature of memories allows the updating of existing memories in light of new information (Forcato, Rodríguez, Pedreira, \& Maldonado, 2010; Hupbach, Gomez, Hardt, \& Nadel, 2007; Rodriguez-Ortiz, De la Cruz, Gutiérrez, \& Bermudez-Rattoni, 2005; Schiller et al., 2010). However, the processes underlying these memory alterations long after initial learning are largely unknown and understanding the dynamics of memory updating is a challenge for current memory research (see Hardt, Einarsson, \& Nader, 2010). One important mechanism that may mediate the modification of apparently stable memories is memory reconsolidation.

For decades, it has been assumed that memories are permanently fixed once they are consolidated (Dudai, 2004; McGaugh, 2000). This view, however, is challenged by studies showing that after reactivation, consolidated and seemingly robust memories may reenter an unstable state, making another phase of stabilization

This article was published Online First March 4, 2013.

Sonja Wichert and Oliver T. Wolf, Institute of Cognitive Neuroscience, Department of Cognitive Psychology, and International Graduate School of Neuroscience, Ruhr-University Bochum, Bochum, Germany; Lars Schwabe, Institute of Cognitive Neuroscience, Department of Cognitive Psychology, Ruhr-University Bochum.

This work was supported by a grant of the Deutsche Forschungsgemeinschaft (DFG; SCHW 1357/5-1). We gratefully acknowledge the assistance of Mathias Hauschild and Deike Müller during data collection. We thank Tobias Otto for his technical assistance.

Correspondence concerning this article should be addressed to Lars Schwabe, Department of Cognitive Psychology, Ruhr-University Bochum, Universitaetsstrasse 150,44780 Bochum, Germany.E-mail:Lars.Schwabe@ rub.de (i.e., reconsolidation) necessary (Milekic \& Alberini, 2002; Nader, Schafe, \& LeDoux, 2000; Suzuki et al., 2004). In this transient plastic state, memories are susceptible to impairments (Debiec, LeDoux, \& Nader, 2002; Duvarci \& Nader, 2004) or the incorporation of new information (Forcato et al., 2010; Hupbach et al., 2007; Rodriguez-Ortiz et al., 2005; Schiller et al., 2010). Such memory modifications after reactivation have by now been demonstrated in a variety of species, ranging from fishes and bees to rodents and humans (for reviews see Hardt et al., 2010; Nader \& Hardt, 2009).

Most reconsolidation studies have used fear conditioning protocols. In rodents, it has been shown that the injection of protein synthesis inhibitors or beta-blockers after fear reactivation may reduce subsequent fear memory (e.g., Nader et al., 2000; Przybyslawski, Roullet, \& Sara, 1999; Sara, 2000). In humans, the administration of beta-blockers during reactivation of a previously conditioned fear memory is also effective in reducing subsequent expression of fear (Kindt, Soeter, \& Vervliet, 2009; Soeter \& Kindt, 2011). Several studies, however, have suggested that memory reconsolidation occurs also in episodic memory and that the reconsolidation process may also be modified by psychological manipulations (Forcato et al., 2007, 2010; Schwabe \& Wolf, 2009; Strange, Kroes, Fan, \& Dolan, 2010; Wichert, Wolf, \& Schwabe, 2011). For example, it has been shown that learning new material after the reactivation of previously learned material may lead to the incorporation of new information into the original memory (i.e., updating; Hupbach et al., 2007). First neuroimaging data point to the potential mechanism that is underlying reconsolidation processes in the human brain and suggest that those brain areas that are recruited during reactivation (particularly the amygdala and the hippocampus) undergo changes in activity that are associated with subsequent changes in memory (Schwabe, Nader, Wolf, Beaudry, \& Pruessner, 2012). 
Memory modifications after reactivation (i.e., during reconsolidation) provide a promising avenue to alter unwanted memories in psychiatric disorders, such as posttraumatic stress disorder (PTSD; Brunet et al., 2008) or addiction (Lee, Milton, \& Everitt, 2006). Given this potential therapeutic relevance of the reconsolidation phenomenon, an important question is whether all memories are at all times susceptible to reconsolidation manipulations. Several boundary conditions that constrain memory reconsolidation have already been identified: the time interval between original learning and reactivation (Wichert et al., 2011; Winters, Tucci, \& DaCostaFurtado, 2009), the duration of the reminder (Pedreira \& Maldonado, 2003), the number of reactivations (Lehmann \& McNamara, 2011), or the context in which reactivation occurs (Hupbach, Hardt, Gomez, \& Nadel, 2008). So far, the main focus has been on the characteristics of the reactivation. Less is known about the nature of postreactivation manipulations that are required to modify the reactivated memory. Rodent data suggest that the impact of pharmacological postreactivation manipulations is dose-dependent: Only a high dose of a protein synthesis inhibitor injected after reactivation led to a substantial decrease in subsequent memory (Duvarci, Nader, \& LeDoux, 2008; Nader et al., 2000). Human data on the nature of postreactivation manipulations, such as new learning, that are needed to alter memories during reconsolidation are completely missing. At the time of initial learning, the degree of (retroactive) interference determines subsequent memory impairment, that is, more intense learning of the interfering material is associated with stronger memory impairments for the initially learned material (Thune \& Underwood, 1943; Twining, 1940; see Lustig \& Hasher, 2001). Although there are obvious differences between interference shortly after initial learning and memory modifications by new learning after reactivation of a consolidated memory, it is tempting to hypothesize that the strength of new learning after reactivation has an impact on subsequent changes in memory.

In the present study, we investigated whether the updating of apparently stable memories after reactivation (i.e., during reconsolidation) depends on the strength of the new encoding. Healthy participants learned new pictures immediately after they had reactivated previously learned pictures. In order to examine whether the strength of new encoding after reactivation affects the extent to which new information is incorporated into the original memory trace, participants learned the new pictures either three times or only once after the reactivation of the previously learned pictures. To control for the effects of new learning alone or reactivation alone, additional groups learned the new pictures without previous reactivation or reactivated the initially learned pictures without subsequently learning new pictures. Another control group neither reactivated the initially learned pictures nor learned new pictures. We hypothesized that the incorporation of new information into the original memory after its reactivation depends on the strength of newly encoded information. We thus expected that new learning after reactivation affects subsequent memory more profoundly when the new pictures are learned three times than when they are learned only once.

\section{Methods}

\section{Participants and Design}

Seventy-two healthy students of the Ruhr-University Bochum (36 men, 36 women; mean age $=24.8$ years, $S E M=0.4$ ) participated in this study. They received either course credits or a moderate monetary compensation for participation. Exclusion criteria comprised current or chronic mental disorder, drug abuse, and current treatment with medication. All participants provided written informed consent for their participation. The study was approved by the local ethics committee.

We used a between-subjects design in which 12 participants (six men, six women) were randomly assigned to each of six experimental groups: reactivation + strong new encoding (Re/strong encoding), no reactivation + strong new encoding (strong encoding), reactivation + weak new encoding (Re/weak encoding), no reactivation + weak new encoding (weak encoding), reactivation + no new encoding $(R e)$, and no reactivation + no new encoding (control; Figure 1).

\section{Stimulus Materials}

The stimulus materials included two sets of 60 pictures taken from the International Affective Picture System (IAPS; Lang, Bradley, \& Cuthbert, 2008). Each set comprised 30 negative and 30 neutral pictures. The pictures of the two sets were matched with respect to emotionality (i.e., emotional valence and emotional arousal), based on the IAPS standard scores for valence and arousal. To ensure that the pictures were indeed experienced as negative and neutral, participants rated each picture with respect to valence and arousal on two $0-100$ scales with the endpoints being neutral versus negative and not arousing versus very arousing, respectively. These ratings confirmed the classification of the pictures as negative and neutral: Negative pictures were rated as significantly more negative than neutral pictures $(M=41.8$, $S E M=2.6$ vs. $M=3.4, S E M=0.7), t(71)=14.7, p<.001$, and

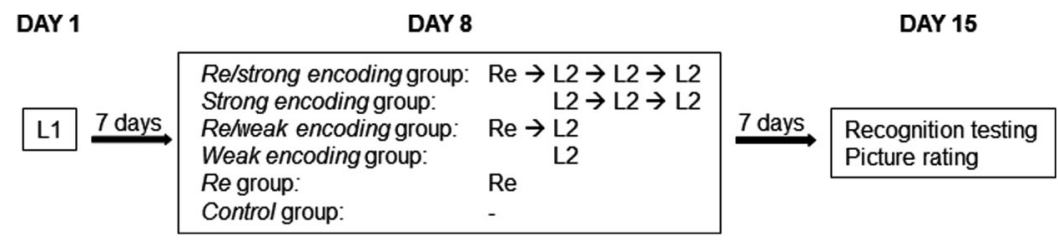

Figure 1. Experimental design. Day 1, initial learning of pictures (L1); Day 8, reactivation of the initially learned pictures (Re) and/or learning of new pictures (L2); Day 15, recognition test for the initially learned pictures, valence and arousal ratings. Day 1 and Day 15 were identical for all participants; Day 8 depended on the group (for details see text). 
were associated with significantly higher emotional arousal than neutral pictures $(M=31.7, S E M=2.5$ vs. $M=4.0, S E M=0.9)$, $t(71)=11.1, p<.001$.

\section{Procedure}

Testing took place on three experimental days: Day 1, initial learning (L1); Day 8, reactivation (react) and/or learning new material either once or three times (weak/strong new encoding); and Day 15, recognition testing, valence and arousal rating (see Figure 1).

On Day 1, all participants saw a set of 60 negative and neutral pictures (L1) in randomized order on a computer screen. Each picture was presented for $2 \mathrm{~s}$, and participants were instructed to memorize the pictures. To control for potential group differences in encoding, participants were asked to recall as many pictures as possible in a free recall test immediately after picture presentation. Participants verbally described the pictures they remembered, and the experimenter checked the mentioned pictures on a list. There was no time limit for the free recall test. The learning session took about $20 \mathrm{~min}$.

The procedure on Day 8, 7 days after Day 1, depended on the experimental group. Participants in the three reactivation groups (Re/strong encoding, Re/weak encoding, $R e$ ) were asked to recall as many pictures from Day 1 as possible. Participants verbally described the pictures they remembered, and the experimenter checked the mentioned pictures on a list to control for potential group differences in memory reactivation. As in earlier studies (Hupbach et al., 2008; Wichert et al., 2011), reactivation took place in the same spatial context as the learning session on Day 1. Immediately after reactivation, participants in the two reactivation/ new learning groups (Re/strong encoding, Re/weak encoding) learned a second picture set of 60 negative and neutral pictures (L2) following the same procedure as during learning of the first picture set on Day 1. In order to assess the effect of new encoding strength on later memory performance, participants learned the second picture set either once (Re/weak encoding) or three times (Re/strong encoding) after reactivation. In the strong encoding condition, participants completed an immediate free recall test after each of the three picture presentations; such repeated free recall tests are known to result in particular strong memories (Karpicke \& Roediger, 2008). To control for the effects of new learning alone, two additional groups learned the second picture set, either once (weak encoding) or three times (strong encoding), without prior reactivation of the first picture set. Another group omitted Day 8 completely (i.e., did not reactivate picture Set 1 and did not learn new pictures; control group). This group served to control for the mere passage of time after initial learning.

On Day 15, 7 days after Day 8, all participants completed a recognition test for the pictures they had learned on Day 1. One hundred eighty pictures were shown, including the pictures that were learned on Day 1 (Set 1), the pictures that were learned by the new learning groups on Day 8 (Set 2), and another Set of 60 neutral and negative pictures that had not been shown before (Set $3)$. Pictures were presented in randomized order and participants were asked to indicate for each picture whether they had seen the picture on Day 1 by pressing a yes or a no button on a keyboard. Recognition testing took about $20 \mathrm{~min}$. Afterward, participants gave valence and arousal ratings for picture Sets 1 and 2 by clicking on valence and arousal scales shown under the picture (see above). Each picture was presented until participants finished their ratings; valence and arousal ratings took about $10 \mathrm{~min}$.

Memory performance in the recognition test on Day 15 was expressed as d-prime (memory sensitivity), percentage of hits (correctly identified pictures from Set 1), and percentage of false alarms (incorrectly identified pictures from picture Sets 2 or 3). For each of the three parameters, we conducted separate Emotionality (negative, neutral) $\times$ Group (Re/strong encoding, Re/weak encoding, strong encoding, weak encoding, Re, and control) ANOVAs, followed by least significant difference (LSD) post hoc tests if indicated. In addition, we performed Emotionality $\times$ Reactivation (yes vs. no) $\times$ New encoding strength (no new encoding, weak new encoding, strong new encoding) ANOVAs in order to directly test the proposed reactivation $\times$ new encoding strength interaction.

\section{Results}

\section{Initial Learning on Day 1}

After initial learning on Day 1, participants remembered on average 22.9 pictures $(S E M=1.5)$. Negative pictures were better recalled than neutral pictures $(M=14.7, S E M=1.5$ vs. $M=8.2$, $S E M=0.4$; main effect of Emotionality, $F(1,66)=17.10, p<$ $.001, \eta^{2}=.21$ ). Importantly, participants' learning performance did not differ between the six groups (main effect of Group and Group $\times$ Emotionality interaction), both $F(1,66)<$ 1.15 , both $p s>.34$, which rules out any differences in encoding of picture Set 1 .

\section{Memory Reactivation and New Learning on Day 8}

During memory reactivation on Day 8, participants in the three reactivation groups (Re/strong encoding, Re/weak encoding, and $R e)$ recalled on average 15.5 pictures $(S E M=0.9)$. Negative pictures were better recalled than neutral pictures $(M=9.9$, $S E M=0.5$ vs. $M=5.6, S E M=0.5$; main effect of Emotionality), $F(1,33)=112.59, p<.001, \eta^{2}=.77$. Groups did not differ in the number of pictures that were recalled during reactivation (main effect of Group and Group $\times$ Emotionality interaction), both $F(1$, $33)<1.10$, both $p$ s $>.34$, thus ruling out group differences in the initial consolidation of picture Set 1 .

After new learning on Day 8, participants in the four new learning groups (Re/strong encoding, Re/weak encoding, strong encoding, and weak encoding) remembered on average 32.6 $(S E M=1.8)$ pictures of Set 2; negative pictures were again better remembered than neutral pictures $(M=18.5, S E M=0.7$ vs. $M=$ $14.1, S E M=0.6$; main effect of Emotionality), $F(1,44)=59.71$, $p<.001, \eta^{2}=.58$. There was, however, a striking difference in the memory performance of participants in the strong and weak new encoding groups (main effect of Group), $F(1,44)=20.80$, $p<.001, \eta^{2}=.59$. At the end of the new learning session, participants in the strong new encoding groups remembered almost twice as many pictures as participants in the weak new encoding groups $(M=41.8, S E M=1.8$ vs. $M=23.3, S E M=1.5$; LSD post hoc tests, $p \mathrm{~s}<.001$ ), indicating that learning the new pictures three times resulted indeed in stronger memory compared with learning them only once. Neutral pictures benefited particularly 
from learning three times (Group $\times$ Emotionality interaction), $F(1$, 44) $=6.11, p=.001, \eta^{2}=.29$, although the beneficial effect of repeated (new) learning was found for neutral (strong vs. weak encoding: $M=8.4, S E M=0.7$ vs. $M=19.7, S E M=1.0), F(1$, $46)=77.31, p<.001, \eta^{2}=.63$, and negative pictures $(M=14.9$, $S E M=1.0$ vs. $M=22.0, S E M=0.8), F(1,46)=29.63, p<.001$, $\eta^{2}=.39$. This may be due to the fact that negative stimuli are already well remembered after only a single encoding trial. Participants who reactivated the initially learned pictures before learning new pictures (Re/strong encoding, Re/weak encoding) and participants who did not reactivate the old memory before new learning (strong encoding, weak encoding) did not differ in their learning performance, neither after learning the new pictures once nor after learning them three times (LSD post hoc tests, both $p \mathrm{~s}>.55)$.

\section{Memory Performance on Day 15}

Memory sensitivity (d-prime). In order to assess the impact of new learning after reactivation on subsequent memory performance, participants completed a recognition test 1 week after reactivation and/or new learning. Memory performance was expressed as d-prime, a measure that takes both correctly identified pictures from picture Set 1 (hits) and incorrectly identified pictures from the picture Sets 2 and 3 (false alarms) into account (Wickens, 2002).

An Emotionality $\times$ Group ANOVA on d-prime revealed a significant main effect of group, $F(1,66)=20.77, p<.001, \eta^{2}=$ .61. Learning new pictures on Day 8 reduced memory performance on Day 15, that is, those groups that learned new pictures on Day 8 were impaired relative to those who did not learn new pictures (LSD post hoc tests, all $p$ s $<.001$ ). Importantly, this memory impairment was most pronounced in participants who learned the new picture set three times after reactivation (Re/strong encoding vs. all other groups; LSD post hoc tests, all $p$ s $<$.007; Figure 2). Moreover, the reactivation of the initially learned pictures before new learning impaired subsequent memory when participants learned the new pictures three times after reactivation (Re/strong encoding vs. strong encoding; LSD post hoc test, $p<.007)$, but not when participants learned the new pictures only once (Re/weak encoding vs. weak encoding; LSD post hoc test, $p=.53$ ). Accordingly, a Reactivation (yes vs. no) $\times$ New encoding strength (no new encoding, weak new encoding, strong new encoding) $\times$ Emotionality ANOVA revealed, in addition to a main effect of New encoding strength, $F(2,66)=47.72, p<.001, \eta^{2}=.59$, a significant Reactivation $\times$ New encoding strength interaction, $F(2$, $66)=3.79, p=.028, \eta^{2}=.10$, showing that memory reactivation before new learning potentiated the subsequent memory impairment in the strong new encoding condition, $F(1,22)=3.88, p=$ $.05, \eta^{2}=.15$, but not in the weak new encoding condition, $F(1$, 22) $=1.19, p=.29, \eta^{2}=.05$.

Memory sensitivity was not modulated by picture emotionality (all main and interaction effects in both ANOVAs: all $p>$ .20).

In order to determine whether the memory impairment in participants who learned the new pictures three times after reactivation was owing to a decrease in the number of correctly identified pictures from Set 1 (hits) or to an increase in the number of

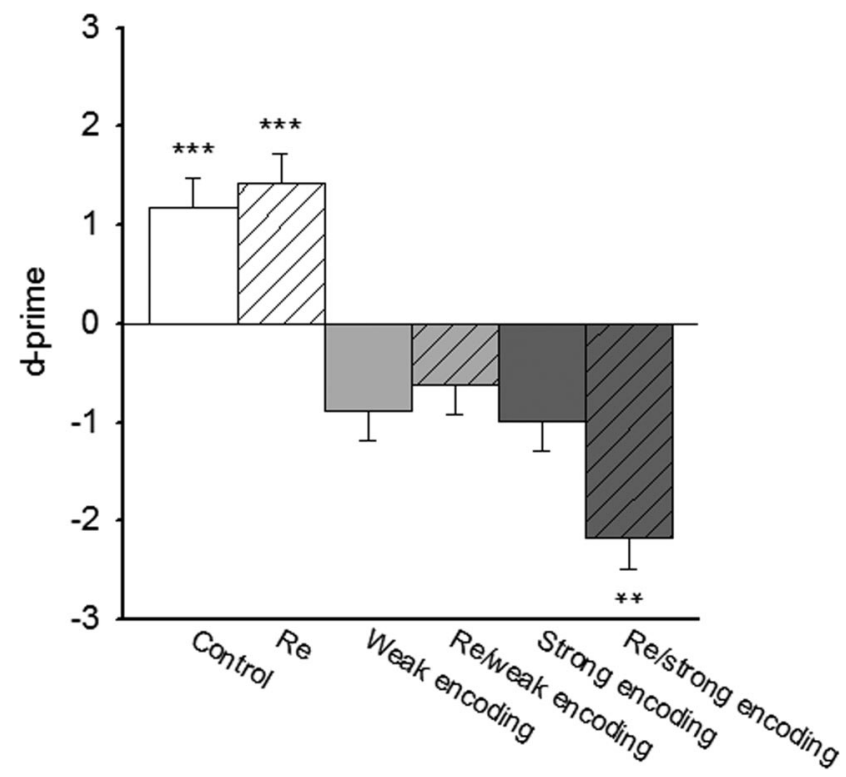

Figure 2. D-prime (i.e., memory sensitivity). Learning new pictures on Day 8 reduced memory sensitivity. However, the impact of new learning on memory sensitivity was strongest when new learning was intense and when it was preceded by memory reactivation. LSD post hoc tests, ${ }^{* *} p \mathrm{~s}<$ .01 compared with all other groups, ${ }^{* * * *} p s<.001$ compared with the new learning groups; data represent mean \pm standard error of the mean.

incorrectly identified pictures from Set 2 or Set 3 (false alarms), we conducted separate analyses for hits and false alarms.

Correctly identified pictures (hits). During recognition testing on Day 15, participants correctly identified on average $46.28 \%$ $(S E M=2.0)$ of the pictures from Set 1 ; more negative than neutral pictures were correctly identified as being from Set $1(M=55.5$, $S E M=1.6$ vs. $M=36.9, S E M=1.9$, main effect of Emotionality) $F(1,66)=110.45, p<.001, \eta^{2}=.63$.

An Emotionality $\times$ Group ANOVA on the percentage of hits revealed a significant main effect of group, $F(1,66)=11.49, p<$ $.001, \eta^{2}=.47$ : The learning of new pictures on Day 8 reduced memory performance on Day 15 irrespective of whether the Set 1 pictures were reactivated before learning or not (LSD post hoc tests, all $p \mathrm{~s}<.01$; Figure 3). Similarly, in a New encoding strength $\times$ Reactivation $\times$ Emotionality ANOVA, we obtained a significant effect of encoding strength, $F(2,66)=27.12, p<.001$, $\eta^{2}=.65$, but no significant Reactivation $\times$ New encoding strength interaction $(p=.27)$.

More negative than neutral pictures were recognized correctly as "old" (main effect Emotionality), $F(1,66)=110.45, p<.001$, $\eta^{2}=.63$; there was, however, no significant interaction effect between Emotionality and Group ( $p=.74)$.

In addition to the analysis of the percentage of hits for all pictures, we analyzed in the groups that had reactivated the List 1 pictures on Day 8 (Re, Re/weak encoding, Re/strong encoding) and also the percentage of hits for only those List 1 pictures that were actually recalled during memory reactivation with a New encoding strength $\times$ Emotionality ANOVA. This analysis yielded a significant effect of New encoding strength, $F(2,33)=6.86, p=.003$, $\eta^{2}=.29$, showing that participants that did not learn new pictures after reactivation recognized virtually all of the pictures they had 


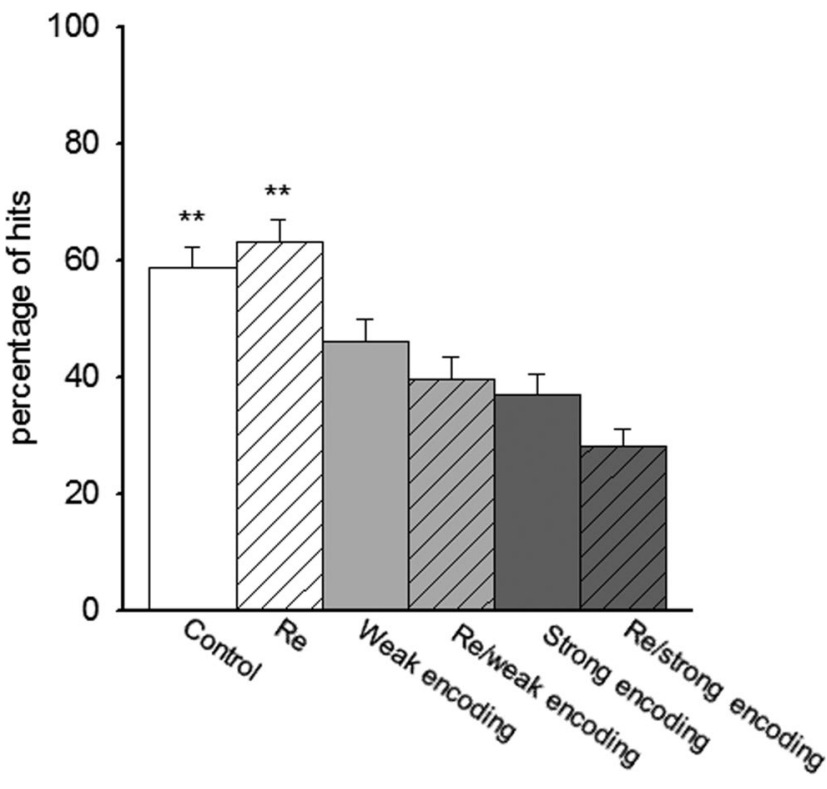

Figure 3. Percentage of hits (i.e., correctly identified pictures from Set 1). Hits were reduced in participants who learned new pictures on Day 8. However, this effect was not dependent on the memory reactivation before new learning. LSD post hoc tests, ${ }^{* * *} p s<.01$ compared with the new learning groups; data represent mean \pm standard error of the mean.

recalled on Day 8 (percentage: $M=94.1, S E M=0.6$ ), whereas participants that learned new pictures once $(M=68.5, S E M=0.7)$ or three times after memory reactivation $(M=71.5, S E M=0.7)$ remembered significantly less of the reactivated pictures on Day 15 (Re vs. Re/strong encoding and Re/weak encoding: both $p<$ .005 ; Re/strong encoding vs. Re/weak encoding: $p=.87$ ). Memory was again stronger for reactivated negative stimuli (main effect Emotionality), $F(1,33)=13.33, p<.001, \eta^{2}=.29$, and memory performance for reactivated neutral pictures was particularly affected by new learning after reactivation (New encoding strength $\times$ Emotionality interaction), $F(2,33)=3.29, p=.05, \eta^{2}=.17$. At this point, it is to be noted that the impact of new encoding strength on memory for only those pictures that were reactivated on Day 8 is only of limited value within the context of memory reconsolidation because in this analysis it is not possible to assess whether the observed memory impairment is reactivation-dependent, that is, it is not possible to disentangle possible reconsolidation effects from mere retroactive interference effects.

Incorrectly identified pictures (false alarms). Participants incorrectly identified on average $15.9 \%($ SEM $=1.5)$ of the pictures from Set 2 or Set 3 as being from Set 1. These false alarms included more negative than neutral pictures $(M=17.9, S E M=$ 1.3 vs. $M=13.9, S E M=1.3$; main effect of Emotionality), $F(1$, 66) $=16.66, p<.001, \eta^{2}=.20$.

An Emotionality $\times$ Group ANOVA on the percentage of false alarms revealed a significant main effect of group, $F(1,66)=$ 8.19, $p<.001, \eta^{2}=.38$. Participants who learned new pictures showed significantly more false alarms compared with participants who did not learn new pictures (LSD post hoc tests, all $p \mathrm{~s}<.03$ ). Importantly, false alarms occurred most often in participants who learned the new pictures three times after reactivation (Re/strong encoding vs. all other groups, LSD post hoc tests, all $p \mathrm{~s}<.08$; Figure 4). Moreover, reactivation before new learning increased the false alarm rate only when the new pictures were learned three times (Re/strong encoding vs. strong encoding, LSD post hoc test, $p<.013$ ), but not when they were learned only once (Re/weak encoding vs. weak encoding, LSD post hoc test, $p=.17$ ). Accordingly, a Reactivation (yes vs. no) $\times$ New encoding strength (no new encoding, weak new encoding, strong new encoding) $\times$ Emotionality ANOVA yielded, in addition to a main effect of New encoding strength, $F(2,66)=18.84, p<.001, \eta^{2}=.36$, a significant Reactivation $\times$ New encoding strength interaction, $F(2$, $66)=3.62, p=.032, \eta^{2}=.10$, showing that memory reactivation before new learning potentiated the false alarm rate in the strong new encoding condition, $F(1,22)=3.84, p=.05, \eta^{2}=.15$, but not in the weak new encoding condition, $F(1,22)=1.41, p=.25$, $\eta^{2}=.06$.

There were more false alarms for negative than for neutral pictures, $F(1,66)=17.52, p<.001, \eta^{2}=.21$, however, the emotionality of the pictures did not modulate the group effect ( $p=$ $.19)$.

Intrusions from Sets 2 and 3. In a next step, we analyzed whether false alarms during recognition testing on Day 15 were mainly due to incorrectly "recognized" pictures from the picture set that was learned after the reactivation of picture Set 1 (Set 2) or from the pictures that participants had never seen before (Set 3). As shown in Figure 5A, the pictures that were incorrectly identified as seen on Day 1 were mainly pictures from Set 2, which were presented after the reactivation of picture Set 1, $t(71)=8.5, p<$ .001. Importantly, intrusions from Set 2 were more frequent when

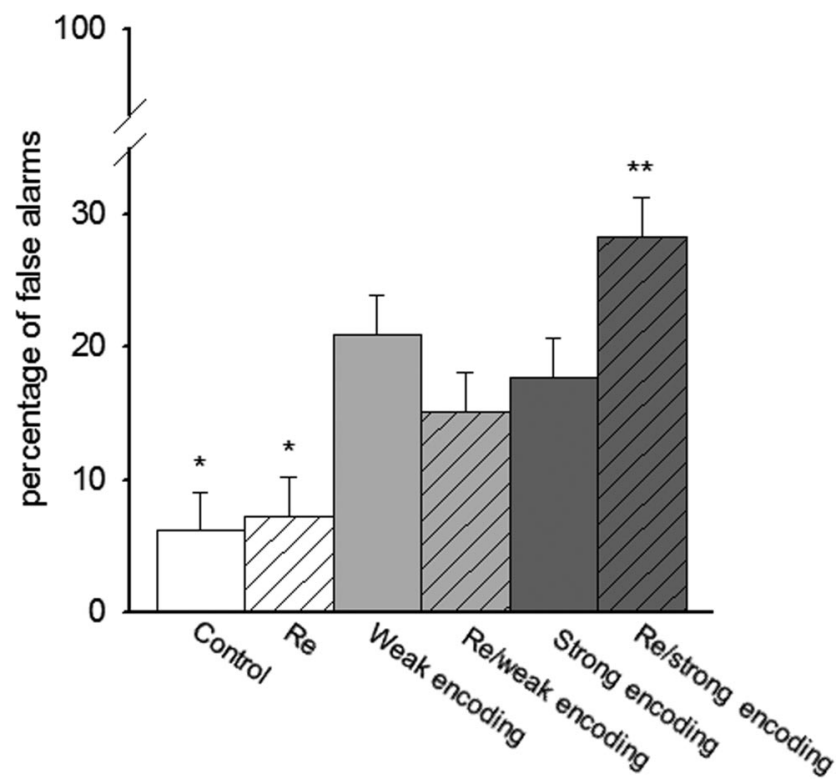

Figure 4. Percentage of false alarms (i.e., incorrectly identified pictures from Set 2 or Set 3). Learning new pictures on Day 8 increased the percentage of false alarms. However, the impact of new learning on false alarms was strongest when new encoding was strong and when it was preceded by memory reactivation. LSD post hoc tests, ${ }^{*} p s<.05$ compared with the new encoding groups, ${ }^{* *} p \mathrm{~s}<.01$ compared with all other groups except to the weak encoding group (LSD post hoc test, $p=.08$ ); data represent mean \pm standard error of the mean. 

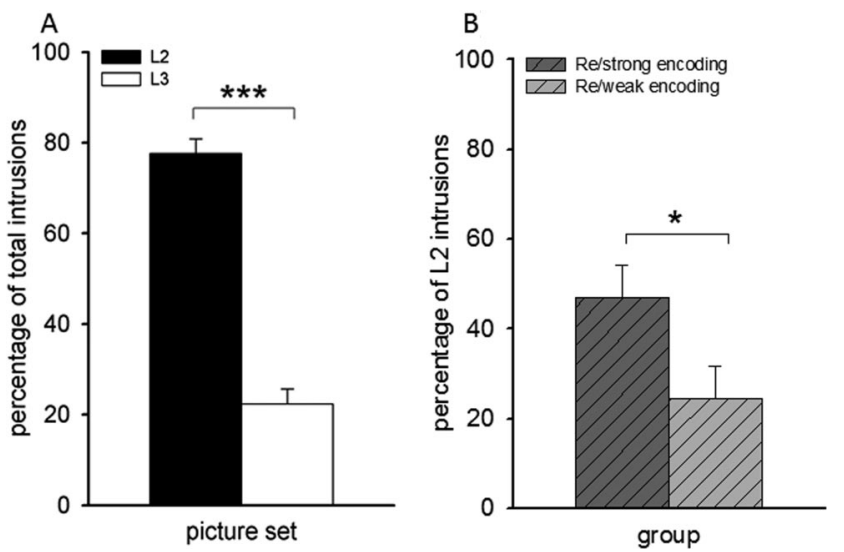

Figure 5. Intrusions from pictures that were learned after reactivation (Set 2) and pictures that have never been learned before (Set 3). (A) In the four new learning groups, intrusions from Set 2 were more frequent than intrusions from Set 3. (B) Intrusions from Set 2 (relative to all pictures that were learned on Day 8) were more frequent when the new pictures were learned three times after reactivation. ${ }^{*} p<.05$, ${ }^{* * *} p<.001$; data represent mean \pm standard error of the mean.

the new pictures were learned three times after reactivation than when they were learned only once after reactivation, $F(1,22)=$ $4.79, p=.04, \eta^{2}=.18$ (Figure 5B). Negative pictures from Set 2 were more often incorrectly recognized as being from Set 1 than neutral pictures $(M=16.7, S E M=0.6$ vs. $M=11.1, S E M=0.7)$, $t(71)=10.7, p<.001$.

\section{Discussion}

The present study examined whether the updating of consolidated memories after reactivation (i.e., during reconsolidation) depends on the strength of new learning. Therefore, participants learned new pictures either three times or only once after the reactivation of previously learned pictures. Our results show that the original memory was altered only when the new information was learned three times but not when it was learned once after reactivation. These findings suggest that the strength of new learning after reactivation is a critical factor in memory updating. Moreover, our finding that strong new encoding led to a significantly more pronounced subsequent memory impairment for the initially learned material when participants reactivated the original memory before new learning than when they did not reactivate the original memory provides further support for the concept of memory reconsolidation (Nader \& Hardt, 2009).

Our findings are in line with previous studies showing that a consolidated episodic memory may be updated by the incorporation of new information during reconsolidation (Forcato et al., 2010; Hupbach et al., 2007). When asked to identify the pictures shown on Day 1 in a recognition test, participants who learned new pictures after the reactivation of the initially learned pictures, incorrectly identified pictures from Day 8 as being from Day 1. Importantly, however, the initially learned pictures did not affect memory for the newly learned pictures in the immediate free recall test for the new pictures on Day 8, suggesting that it was indeed the reactivated original memory and not the memory for the new information that was modified. These findings support the idea that the main purpose of memory reconsolidation is memory updating (Nadel \& Moscovitch, 1997).

Previous studies reported alterations in subsequent memory when new information was learned several times after reactivation (Wichert et al., 2011; Wichert, Wolf, \& Schwabe, 2013) when participants saw real objects after reactivation (Hupbach et al., 2007, 2008; Hupach, Gomez, \& Nadel, 2009), or when participants saw only relatively little new information after reactivation (Forcato et al., 2007, 2010). All these manipulations have most likely led to relatively strong memories for each of the newly encoded items. The present study is the first that investigated explicitly the impact of new encoding strength on memory reconsolidation and shows that reconsolidation effects (i.e., the updating of the original memory) are indeed influenced by the strength of the new encoding. Why does the strength of new learning affect the modification of an original memory after its reactivation? New information that is learned three times after reactivation may be considered as being of higher relevance than new information that is learned only once and may therefore be more likely to be incorporated into the original memory (Rescorla \& Wagner, 1972; Rodriguez-Ortiz et al., 2005). Emotionality is another factor that determines the relevance of information: Emotionally arousing information is more relevant for survival than neutral information. It is therefore not surprising that negative pictures were generally better remembered than neutral pictures in the present study. Even though in our study the reconsolidation effect itself was not emotionality-dependent, we found that more negative than neutral pictures from Set 2 were incorporated into the original memory, thus providing further evidence that more relevant information is more likely to be integrated into a reactivated memory trace. Moreover, both increased encoding strength and increased emotionality are associated with increased memory strength (Suzuki et al., 2004), so it might actually be the strength of new memories that determines their relevance. Accordingly, we suggest that the strength of new memories is a critical factor for the incorporation of new information into an original memory.

Although there are parallels between memory reconsolidation and retroactive interference at first glance (e.g., the impairing effect of new learning) and although an influence of new learning strength similar to the one reported here has been shown for (retroactive) interference (Thune \& Underwood, 1943; Twining, 1940), there are important differences between memory reconsolidation and retroactive interference, for example, in the experimental design as well as in the observed effects. In the retroactive interference paradigm, learning of the new information has to take place shortly after initial learning in order to be effective. In the reconsolidation paradigm, however, new learning may occur long after initial learning because memory reactivation makes the consolidated memory susceptible to modifications. Memory reactivation is necessary for memory modifications during reconsolidation, which is also confirmed by our data showing that intense new learning led only to a pronounced memory alteration when it was preceded by the reactivation of the original memory. Moreover, reconsolidation effects go beyond the effects of classical interference in that new learning after reactivation may impair (Forcato et al., 2007; Wichert et al., 2011) or update (Hupbach et al., 2007; Schiller et al., 2010) memory, whereas interference after initial learning results in an impairment of the original memory. 
Just as new learning after reactivation can alter memory, exposure to misleading information between initial learning and later recall can result in the alteration of memory, which is known as misinformation effect (Loftus, 2005; Loftus \& Pickrell, 1995). In the misinformation paradigm, participants receive information about an event and are later asked misleading questions about that event. In a subsequent memory test for the event, participants often report the misinformation instead of the initial information, indicating that the misleading information has been incorporated into the original memory (Loftus, 1975). Can memory reconsolidation account for misinformation effects? In the misinformation paradigm, the misleading questions are directly related to the original event, making it likely that the questions reactivate the original memory. As a consequence, the reactivated original memory for the event is susceptible to modifications such as the incorporation of misleading information. Thus, reconsolidation may actually be the mechanism that is underlying misinformation effects. Interestingly, the likelihood of recalling the misleading information is higher for questions that contain true statements about the original event than for questions that contain false statements about the original event (Loftus, 1975). Given that true statements may be more relevant than false ones, these findings are in line with our assumption that the relevance of the new information determines the incorporation of new information into an original memory.

Taken together, we examined whether the updating of episodic memories during reconsolidation depends on the strength of new encoding after memory reactivation. Our results show that new information was incorporated into an original memory only when the new information was learned three times but not when it was learned only once after reactivation. These findings indicate that the strength of new learning after reactivation is critical for the updating of original memories. The importance of new encoding strength for memory updating may be relevant in the context of therapeutic interventions that focus on the alteration of unwanted memories after memory reactivation, for example, in PTSD.

\section{References}

Brunet, A., Orr, S. P., Tremblay, J., Robertson, K., Nader, K., \& Pitman, R. K. (2008). Effect of post-retrieval propranolol on psychophysiologic responding during subsequent script-driven traumatic imagery in posttraumatic stress disorder. Journal of Psychiatric Research, 42, 503-506. doi:10.1016/j.jpsychires.2007.05.006

Debiec, J., LeDoux, J. E., \& Nader, K. (2002). Cellular and systems reconsolidation in the hippocampus. Neuron, 36, 527-538. doi:10.1016/ S0896-6273(02)01001-2

Dudai, Y. (2004). The neurobiology of consolidation, or, how stable is the engram? Annual Review of Psychology, 55, 51-86. doi:10.1146/annurev .psych.55.090902.142050

Duvarci, S., \& Nader, K. (2004). Characterization of fear memory reconsolidation. The Journal of Neuroscience, 24, 9269-9275. doi:10.1523/ JNEUROSCI.2971-04.2004

Duvarci, S., Nader, K., \& LeDoux, J. E. (2008). De novo mRNA synthesis is required for both consolidation and reconsolidation of fear memories in the amygdala. Learning \& Memory, 15, 747-755. doi:10.1101/1m .1027208

Forcato, C., Burgos, V. L., Argibay, P. F., Molina, V. A., Pedreira, M. E., \& Maldonado, H. (2007). Reconsolidation of declarative memory in humans. Learning \& Memory, 14, 295-303. doi:10.1101/lm.486107

Forcato, C., Rodríguez, M. L. C., Pedreira, M. E., \& Maldonado, H. (2010). Reconsolidation in humans opens up declarative memory to the entrance of new information. Neurobiology of Learning and Memory, 93, 77-84. doi:10.1016/j.nlm.2009.08.006

Hardt, O., Einarsson, E. Ö., \& Nader, K. (2010). A bridge over troubled water: Reconsolidation as a link between cognitive and neuroscientific memory research traditions. Annual Review of Psychology, 61, 141-167. doi:10.1146/annurev.psych.093008.100455

Hupbach, A., Gomez, R., Hardt, O., \& Nadel, L. (2007). Reconsolidation of episodic memories: A subtle reminder triggers integration of new information. Learning \& Memory, 14, 47-53. doi:10.1101/1m.365707

Hupbach, A., Gomez, R., \& Nadel, L. (2009). Episodic memory reconsolidation: Updating or source confusion? Memory, 17, 502-510. doi: 10.1080/09658210902882399

Hupbach, A., Hardt, O., Gomez, R., \& Nadel, L. (2008). The dynamics of memory: Context-dependent updating. Learning \& Memory, 15, 574 579. doi:10.1101/1m.1022308

Karpicke, J. D., \& Roediger, H. K. (2008). The critical importance of retrieval for learning. Science, 319, 966-968. doi:10.1126/science .1152408

Kindt, M., Soeter, M., \& Vervliet, B. (2009). Beyond extinction: Erasing human fear responses and preventing the return of fear. Nature Neuroscience, 12, 256-258. doi:10.1038/nn.2271

Lang, P. J., Bradley, M. M., \& Cuthbert, B. N. (2008). International affective picture system (IAPS): Affective ratings of pictures and instruction manual. (Technical Report A-8). University of Florida, Gainesville, FL.

Lee, J. L. C., Milton, A. L., \& Everitt, B. J. (2006). Cue-induced cocaine seeking and relapse are reduced by disruption of drug memory reconsolidation. The Journal of Neuroscience, 26, 5881-5887. doi:10.1523/ JNEUROSCI.0323-06.2006

Lehmann, H., \& McNamara, K. C. (2011). Repeatedly reactivated memories become more resistant to hippocampal damage. Learning \& Memory, 18, 132-135. doi:10.1101/lm.2000811

Loftus, E. F. (1975). Leading questions and the eyewitness report. Cognitive Psychology, 7, 560-572. doi:10.1016/0010-0285(75)90023-7

Loftus, E. F. (2005). Planting misinformation in the human mind: A 30-year investigation of the malleability of memory. Learning \& Memory, 12, 361-366. doi:10.1101/1m.94705

Loftus, E. F., \& Pickrell, J. E. (1995). The formation of false memories. Psychiatric Annals, 25, 720-725.

Lustig, C., \& Hasher, L. (2001). Implicit memory is not immune to interference. Psychological Bulletin, 127, 618-628. doi:10.1037/00332909.127.5.618

McGaugh, J. L. (2000). Memory-A century of consolidation. Science, 287, 248-251. doi:10.1126/science.287.5451.248

Milekic, M. H., \& Alberini, C. M. (2002). Temporally graded requirement for protein synthesis following memory reactivation. Neuron, 36, 521525. doi:10.1016/S0896-6273(02)00976-5

Nadel, L., \& Moscovitch, M. (1997). Memory consolidation, retrograde amnesia and the hippocampal complex. Current Opinion in Neurobiology, 7, 217-227. doi:10.1016/S0959-4388(97)80010-4

Nader, K., \& Hardt, O. (2009). A single standard for memory: The case for reconsolidation. Nature Reviews Neuroscience, 10, 224-234. doi: $10.1038 / \mathrm{nrn} 2590$

Nader, K., Schafe, G. E., \& LeDoux, J. E. (2000). Fear memories require protein synthesis in the amygdala for reconsolidation after retrieval. Nature, 406, 722-726. doi:10.1038/35021052

Pedreira, M. E., \& Maldonado, H. (2003). Protein synthesis subserves reconsolidation or extinction depending on reminder duration. Neuron, 38, 863-869. doi:10.1016/S0896-6273(03)00352-0

Przybyslawski, J., Roullet, P., \& Sara, S. J. (1999). Attenuation of emotional and nonemotional memories after their reactivation: Role of $\beta$ adrenergic receptors. The Journal of Neuroscience, 19, 6623-6628.

Rescorla, R. A., \& Wagner, A. R. (1972). A theory of pavlovian conditioning: Variations in the effectiveness of reinforcement and nonrein- 
forcement. In A. H. Black \& W. F. Prokasy (Eds.), Classical conditioning II: Current research and theory (pp. 29-37). New York, NY: Appleton-Century Crofts.

Rodriguez-Ortiz, C. J., De la Cruz, V., Gutiérrez, R., \& Bermudez-Rattoni, F. (2005). Protein synthesis underlies post-retrieval memory consolidation to a restricted degree only when updated information is obtained. Learning \& Memory, 12, 533-537. doi:10.1101/lm.94505

Sara, S. J. (2000). Retrieval and reconsolidation: Toward a neurobiology of remembering. Learning \& Memory, 7, 73-84. doi:10.1101/lm.7.2.73

Schiller, D., Monfils, M-H., Raio, C. M., Johnson, D. C., LeDoux, J. E., \& Phelps, E. A. (2010). Preventing the return of fear in humans using reconsolidation update mechanisms. Nature, 463, 49-53. doi: 10.1038/nature08637

Schwabe, L., Nader, K., Wolf, O. T., Beaudry, T., \& Pruessner, J. C. (2012). Neural signature of reconsolidation impairments by propranolol in humans. Biological Psychiatry, 71, 380-386. doi:10.1016/j.biopsych .2011.10.028

Schwabe, L., \& Wolf, O. T. (2009). New episodic learning interferes with the reconsolidation of autobiographical memories. PLOS ONE, 4, doi: 10.1371/journal.pone.0007519

Soeter, M., \& Kindt, M. (2011). Disrupting reconsolidation: Pharmacological and behavioral manipulations. Learning \& Memory, 18, 357-366. doi:10.1101/lm.2148511

Strange, B. A., Kroes, M. C. W., Fan, J. E., \& Dolan, R. J. (2010). Emotion causes targeted forgetting of established memories. Frontiers in Behavioral Neuroscience, 4, 1-13. doi:10.3389/fnbeh.2010.00175
Suzuki, A., Josselyn, S. A., Frankland, P. W., Masushige, S., Silva, A. J., \& Kida, S. (2004). Memory reconsolidation and extinction have distinct temporal and biochemical signatures. The Journal of Neuroscience, 24, 4787-4795. doi:10.1523/JNEUROSCI.5491-03.2004

Thune, L. E., \& Underwood, B. J. (1943). Retroactive inhibition as a function of degree of interpolated learning. Journal of Experimental Psychology, 32, 185-200. doi:10.1037/h0055133

Twining, P. E. (1940). The relative importance of intervening activity and lapse of time in production of forgetting. Journal of Experimental Psychology, 26, 483-501. doi:10.1037/h0055340

Wichert, S., Wolf, O. T., \& Schwabe, L. (2011). Reactivation, interference, and reconsolidation: Are recent and remote memories likewise susceptible? Behavioral Neuroscience, 125, 699-704. doi:10.1037/a0025235

Wichert, S., Wolf, O. T., \& Schwabe, L. (2013). Changing memories after reactivation: A one-time opportunity? Neurobiology of Learning and Memory, 99, 38-49.

Wickens, T. D. (2002). Elementary signal detection theory. Oxford: Oxford University Press.

Winters, B. D., Tucci, M., \& DaCosta-Furtado, M. (2009). Older and stronger object memories are selectively destabilized by reactivation in the presence of new information. Learning \& Memory, 16, 545-553. doi:10.1101/lm.1509909

Received July 26, 2012

Revision received November 29, 2012

Accepted January 28, 2013 